\title{
Topological Models for Extensional Partial Set Theory
}

\author{
Roland Hinnion and Thierry Libert
}

\begin{abstract}
We state the consistency problem of extensional partial set theory and prove two complementary results toward a definitive solution. The proof of one of our results makes use of an extension of the topological construction that was originally applied in the paraconsistent case.
\end{abstract}

\section{Introduction}

Since Gilmore's seminal work on partial set theory [1], different versions of set theory based on positive comprehension schemes have been studied. This paper is concerned with one of these in particular, namely, the simple version of partial set theory with extensionality, the consistency problem of which is still unsolved, surprisingly. We describe in this first section that theory, what is known about it, and particularly the insights the present paper brings into its consistency problem. We shall refer the reader interested in the historical aspect of the subject to [3].

Consider the language of set theory $\mathscr{L}$ defined by the following rules of formation in first-order predicate calculus without equality - the axioms for equality will be explicitly stated below:

(1) $\mathrm{T}$ (the 'true') and $\perp$ (the 'false') are (atomic) formulas;

(2) if $x, y$ are variables, $x \in y$ and $x \notin y$ are (atomic) formulas;

(3) if $x, y$ are variables, $x=y$ and $x \neq y$ are (atomic) formulas;

(4) if $\varphi, \psi$ are formulas, so are $\varphi \wedge \psi$ and $\varphi \vee \psi$;

(5) if $\varphi$ is a formula and $x$ a variable, then $\forall x \varphi$ and $\exists x \varphi$ are formulas;

(6) if $\varphi$ is a formula, so is $\neg \varphi$;

The positive fragment, denoted by $\mathscr{L}^{+}$, is obtained without using rule (6). Note that $\notin$ and $\neq$ are considered here as primitive symbols, not as abbreviations of the

Received May 29, 2006; accepted June 15, 2007; printed December 3, 2007 2000 Mathematics Subject Classification: Primary, 03E35, 03E70, 03E99, 03C30

Keywords: partial set theory, paraconsistent set theory, extensionality

(c) 2007 by University of Notre Dame $\quad 10.1215 / 00294527-2007-002$ 
classical negation of $\epsilon$ and $=$, respectively. In other words, we are not assuming the additional axioms,

$$
\mathrm{Cl}(\notin): \equiv \forall x \forall y(x \notin y \leftrightarrow \neg(x \in y)) \& \mathrm{Cl}(\neq): \equiv \forall x \forall y(x \neq y \leftrightarrow \neg(x=y)) .
$$

We are, however, going to consider that one and only one of the following dual axioms holds at the same time:

$$
\begin{aligned}
& \mathrm{Pt}: \equiv \forall x \forall y(\neg(x \in y \wedge x \notin y) \wedge \neg(x=y \wedge x \neq y)) \quad \text { [the partial case] } \\
& \text { or } \\
& \mathrm{Pd}: \equiv \forall x \forall y((x \in y \vee x \notin y) \wedge(x=y \vee x \neq y)) \quad \text { [the paradoxical case] }
\end{aligned}
$$

Thereupon, we define the weak negation $\bar{\varphi}$ of an $\mathscr{L}^{+}$-formula $\varphi$ as follows:

$$
\begin{array}{cl}
\bar{\perp} \text { is } \top, & \bar{\top} \text { is } \perp, \\
\overline{x \in y} \text { is } x \notin y, & \overline{x \notin y} \text { is } x \in y, \\
\overline{x=y} \text { is } x \neq y, & \overline{x \neq y} \text { is } x=y, \\
\overline{\varphi \wedge \psi} \text { is } \bar{\varphi} \vee \bar{\psi}, & \overline{\varphi \vee \psi} \text { is } \bar{\varphi} \wedge \bar{\psi}, \\
\overline{\forall x \varphi} \text { is } \exists x \bar{\varphi}, & \overline{\exists x \varphi} \text { is } \forall x \bar{\varphi} .
\end{array}
$$

Clearly, $\bar{\varphi}$ is in $\mathscr{L}^{+}$and $\overline{\bar{\varphi}}$ is $\varphi$. And it is easy to see that Pt is equivalent to $\bar{\varphi} \rightarrow \neg \varphi$ for all $\mathscr{L}^{+}$-formulas $\varphi$, whereas $\operatorname{Pd}$ is equivalent to $\neg \varphi \rightarrow \bar{\varphi}$ for all $\mathscr{L}^{+}$-formulas $\varphi$.

As primitive symbol, $=$ is of course intended to be an equality relation, so we would expect both of the following axiom and scheme of axioms to hold:

$$
\operatorname{Refl}: \equiv \forall x(x=x) \& \text { Subst }: \equiv\left[\begin{array}{l}
\text { for any } \mathscr{L} \text {-formula } \varphi \text { and } \varphi_{(y \mid x)}{ }^{1} \\
\forall x \forall y\left(x=y \rightarrow\left(\varphi \leftrightarrow \varphi_{(y \mid x)}\right)\right) .
\end{array}\right.
$$

In what follows, we let Eq stand for Refl + Subst.

In set theory, it is usually required that sets be determined by their (actual) members, so $=$ would also be characterized by the so-called axiom of extensionality:

$$
\text { Ext }: \equiv \forall x \forall y(x=y \rightarrow x=y),
$$

where $x=y$ is an abbreviation for $\forall z((z \in x \leftrightarrow z \in y) \wedge(z \notin x \leftrightarrow z \notin y)){ }^{2}$

At last, what characterizes set theory itself, in its naïve conception at least, is the so-called comprehension scheme, whose natural translation in this context is as follows:

$$
\operatorname{Comp}\left[\mathscr{L}^{+}\right]: \equiv\left[\begin{array}{l}
\text { for any } \mathscr{L}^{+} \text {-formula } \varphi(x, \bar{z}), \\
\forall \bar{z} \exists y \forall x((x \in y \leftrightarrow \varphi(x, \bar{z})) \wedge(x \notin y \leftrightarrow \bar{\varphi}(x, \bar{z}))) .
\end{array}\right.
$$

Now, the introduction of $\notin$ and $\neq$ as primitive symbols, and the presentation above that comes with, can be motivated by the following fact.

Fact On the one hand, $\operatorname{Comp}\left[\mathscr{L}^{+}\right]+\mathrm{Cl}(\notin)$ is known to be inconsistent. On the other hand, $\operatorname{Comp}\left[\mathscr{L}^{+}\right]+\mathrm{Pt} / \mathrm{Pd}$ has been proved to be consistent.

The inconsistency of Comp $\left[\mathscr{L}^{+}\right]+\mathrm{Cl}(\notin)$ is of course Russell's paradox, whereas the consistency of $\mathrm{Eq}+\operatorname{Comp}\left[\mathscr{L}^{+}\right]+$Pt directly follows from Gilmore's result on Partial Set Theory [1]; actually, Gilmore's inductive technique would even enable one to show that $\mathrm{Eq}+\mathrm{Comp}\left[\mathscr{L}^{+}\right]+\mathrm{Pt}+\mathrm{Cl}(\neq)$ is consistent, as one could expect. 
We just mention here that the same technique works, and so the same result holds, with $\mathrm{Pd}$ instead of $\mathrm{Pt}$, and that in both cases the key property is the positivity of the formulas involved in the comprehension scheme. Details and references can be found in [3], and even further in [5] for the Pd case.

The difficulty arises when one requires Ext. The reason is that Ext asserts that $=$ coincides with $\bar{F}$, and this latter is not defined by an $\mathscr{L}^{+}$-formula, so those formulas containing $=$ involved in the comprehension scheme are not actually positive. As a matter of fact, Gilmore himself proved in [1] that his technique is then no longer applicable. ${ }^{3}$ Note, however, that it is possible to show by that technique that $\mathrm{Eq}+\mathrm{Ext}+\operatorname{Comp}\left[\mathscr{L}_{*}^{+}\right]+\mathrm{Pt} / \mathrm{Pd}$ is consistent, where $\mathscr{L}_{*}^{+}$is the fragment of $\mathscr{L}$ obtained without using rule (6) or rule (3); this is due to Brady. Again, we would refer the reader to [3] for a precise account.

The consistency of Eq $+\operatorname{Ext}+\operatorname{Comp}\left[\mathscr{L}^{+}\right]+\mathrm{Pd}$ came out of [2], where topological models are presented. On the other hand, despite the apparent symmetry, the consistency of Eq + Ext + Comp $\left[\mathscr{L}^{+}\right]+\mathrm{Pt}$, which we call the "simple" version of extensional partial theory, has surprisingly resisted all the attempts so far. As this will be made precise in Part I, the symmetry is indeed perfect if we require $\mathrm{Cl}(\neq)$, but the consistency of $\mathrm{Eq}+\mathrm{Ext}+\mathrm{Comp}\left[\mathscr{L}^{+}\right]+\mathrm{Cl}(\neq)$-without even adding $\mathrm{Pd}$ or $\mathrm{Pt}$-is also an open problem.

We only focus on the consistency problem for $\mathrm{Eq}+\mathrm{Ext}+\mathrm{Comp}\left[\mathscr{L}^{+}\right]+\mathrm{Pt}$ in this paper, and the progress we make is twofold:

1. In Part I, by a careful analysis of the symmetry $\mathrm{Pt} / \mathrm{Pd}$, we show the consis-

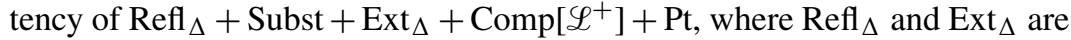
restricted versions of Refl and Ext on classical sets (Theorem I.3).

2. In Part II, we prove the consistency of Eq $+\operatorname{Ext}+\operatorname{Comp}\left[\mathscr{L}_{\Theta}^{+}\right]+\mathrm{Pt}$, where $\mathscr{L}_{\Theta}^{+}$is obtained by replacing the unrestricted quantifiers $\forall$ and $\exists$ in rule (5) by bounded quantifiers of the form $\forall_{\Theta}$ and $\exists_{\Theta}$, which are defined by $\forall_{\Theta} x \varphi: \equiv \forall x\left(\Theta_{x} \rightarrow \varphi\right)$ and $\exists_{\Theta} x \varphi: \equiv \exists x\left(\Theta_{x} \wedge \varphi\right)$, where $\Theta_{x}$ is some $(N+1)$-ary predicate symbol added to the language (Theorem II.10).

For lack of a definitive solution to that consistency problem, these results at least give insights into it. Both of them make use of topological models, and those we describe in Part II are new; the technique we apply here is a generalization of the original construction by inverse limits used in [2].

\section{Part I Symmetry}

Clearly, interchanging $\in$ with $\notin$ and $=$ with $\neq$ preserves Comp $\left[\mathscr{L}^{+}\right]$. Likewise, replacing any atomic formula by its (strong) negation in all instances of $\operatorname{Comp}\left[\mathscr{L}^{+}\right]$ does not affect the veracity of that scheme (duality). But each of these transformations taken individually is rather inappropriate as far as Eq is concerned, in that neither $x \neq y$ nor $\neg(x=y)$ is likely to behave as an equality relation. Combining both of these transformations simultaneously is more natural and effective as we shall see.

We define the adjunct $\widehat{\varphi}$ of an $\mathscr{L}$-formula $\varphi$ as the formula obtained from $\varphi$ by replacing each of its atomic formulas $\psi$ by $\neg \bar{\psi}$. It is easy to see that $\widehat{\varphi}$ is equivalent to $\neg \bar{\varphi}$ for each $\varphi$ in $\mathscr{L}^{+}$, and by definition $\widehat{\neg \varphi}$ is $\neg \widehat{\varphi}$ for all $\varphi$. For example, $\widehat{\mathrm{Pt}}$ is equivalent to $\mathrm{Pd}$, and vice versa. Obviously, $\widehat{\hat{\varphi}}$ is equivalent to $\varphi$ for all $\varphi$. A formula 
$\varphi$ is said to be self-adjunct if $\widehat{\varphi}$ is equivalent to $\varphi$. It is easily seen that all instances of Comp $\left[\mathscr{L}^{+}\right]$are self-adjunct.

On the semantic side, we define the adjunct $\widehat{U}$ of an $\mathscr{L}$-structure $U$ by $|\widehat{U}|:=|U|$, $\in \widehat{u}:=|u|^{2} \backslash \notin u, \notin \widehat{u}:=|u|^{2} \backslash \in u,=\widehat{u}:=|u|^{2} \backslash \neq u, \neq \widehat{u}:=|u|^{2} \backslash=u$. Clearly, $\widehat{\hat{U}}$ is $U$, and it follows from the definitions that $U \models \varphi(\bar{u})$ if and only if $\widehat{U} \models \widehat{\varphi}(\bar{u})$, for any $\mathscr{L}$-formula $\varphi(\bar{x})$ and $\bar{u}$ in $|\mathcal{U}|$. In particular, $\widehat{U}$ models Comp $\left[\mathscr{L}^{+}\right]$if and only if $\mathcal{U}$ does. Note also that $\mathcal{U}$ is self-adjunct (i.e., $\widehat{U}=\mathcal{U}$ ) if and only if $\mathcal{U}$ is classical (i.e., $U \models \mathrm{Pd} \wedge \mathrm{Pt}$, that is, $U \models \mathrm{Cl}(\notin) \wedge \mathrm{Cl}(\neq)$-notice by the way that both $\mathrm{Cl}(\notin)$ and $\mathrm{Cl}(\neq)$ are self-adjunct).

We now turn to the axioms of equality and extensionality. We first observe that $=$ is self-adjunct, whereas $=$ is self-adjunct if and only if $\mathrm{Cl}(\neq)$. Thus, assuming $\dot{u} \models \mathrm{Cl}(\neq)$, we have $u \models \mathrm{Eq}+$ Ext if and only if $\widehat{u} \models \mathrm{Eq}+$ Ext. But, as said in the Introduction, the existence of $\mathcal{U}$ satisfying $\mathrm{Eq}+\mathrm{Ext}+\mathrm{Comp}\left[\mathscr{L}^{+}\right]+\mathrm{Cl}(\neq)$ is unknown. At least, without $\mathrm{Cl}(\neq)$, the following implications hold.

\section{Lemma I.1}

(i) If $\mathcal{U}=\mathrm{Pt}+$ Refl, then $\widehat{u}=\mathrm{Pd}+$ Refl.

(ii) If $\mathcal{U}=\mathrm{Pd}+$ Subst, then $\widehat{u} \models \mathrm{Pt}+$ Subst.

(iii) If $u \models \mathrm{Pt}+$ Ext, then $\widehat{u} \models \mathrm{Pd}+$ Ext.

\section{Proof}

(i) Suppose $U \models \mathrm{Pt}+\operatorname{Refl}$. Then $\widehat{U} \models \widehat{\operatorname{Refl}}$, and as $\widehat{U} \models \forall x(\neg(x \neq x) \rightarrow x=x)$ (Pd case), we get $\widehat{U} \models$ Refl.

(ii) Suppose $u \models P d+$ Subst. Then $\widehat{U} \models \widehat{\text { Subst }}$, and since $\widehat{U} \models \forall x \forall y(x=y$ $\rightarrow \neg(x \neq y))$ (Pt case), we have $\widehat{U} \models \forall x \forall y(x=y \rightarrow(\widehat{\varphi} \leftrightarrow \widehat{\varphi}(y \mid x)))$, for all formulas $\varphi$ in $\mathscr{L}$, and so in particular of the form $\widehat{\psi}$ for any $\mathscr{L}$-formula $\psi$. Hence $\widehat{u} \models$ Subst.

(iii) Suppose $U \models \mathrm{Pt}+$ Ext. Then $\widehat{u} \models \widehat{\mathrm{Ext}}$, and as $\widehat{u} \models \forall x \forall y(\neg(x \neq y)$ $\rightarrow x=y$ ) (Pd case), we get $\widehat{u} \models$ Ext, for we recall that $x=y$ is self-adjunct.

Without requiring $\mathrm{Cl}(\neq)$, the converse of (i), (ii), (iii) above fail. To see that the converse of (ii) does not hold, consider any $U \models \mathrm{Pt}+$ Subst such that $u \vDash u=u \wedge \neg(v=u) \wedge \neg(u \neq v)$ for some $u, v$ in $|U|$, so that $\widehat{u} \models$ $\neg(u \neq u) \wedge v \neq u \wedge u=v$, and thus $\widehat{u} \not$ Subst. For instance, this will be the case for any $u \models P t$ with $|u|>1,=u=\delta_{|u|}$, and $\neq u=\varnothing .{ }^{4}$ A more interesting example will be given by our topological model in Part II.

Now to see that the converse of (i) and (iii) fail, consider any $U \models \mathrm{Pd}+\mathrm{Refl}+$ Ext such that $u \models u \neq u$ for some $u$ in $|U|$, so that $\widehat{u} \models \neg(u=u)$, and thus $\widehat{u} \not \models$ Refl and $\widehat{u} \not \models$ Ext (because $\widehat{U} \models u=u$, obviously). A very interesting example of such a $U$ appeared in [2] and then was further studied in [5], in both of which papers is proved the following.

Fact I.2 There exists $u \models \mathrm{Pd}+\mathrm{Eq}+\operatorname{Ext}+\operatorname{Comp}\left[\mathscr{L}^{+}\right]$such that $u \models$ $\forall x \forall y(x \neq y \leftrightarrow x \neq y)$, where $x \neq y: \equiv \exists z((z \in x \wedge z \notin y) \vee(z \notin x \wedge z \in y))$.

In such a $U$, we have $\mathcal{U} \models \forall x(x \neq x \leftrightarrow \nabla(x))$, where $\nabla(x): \equiv x \neq x \equiv$ $\exists z(z \in x \wedge z \notin x)$ is just the statement asserting that $x$ is not classical (with respect to the Pd case). And as $U \models \operatorname{Comp}\left[\mathscr{L}^{+}\right]$, there does exist $r$ in $|\mathcal{U}|$ such that $u \models \forall x(x \in r \leftrightarrow x \notin x)$ (i.e., $r$ is the Russell set), from which it is easy to see that 
$u \models \nabla(r)$, so that $u \models r \neq r$. As noticed above, it follows that $\widehat{u} \vDash$ Refl and $\widehat{u} \not \models$ Ext. Nevertheless, thanks to the characterization of $\neq$ in $u$, something of Refl and Ext remains in $\widehat{U}$; namely, Refl and Ext are preserved on classical sets, that is, those sets $x$ satisfying $\Delta(x): \equiv \forall z(z \in x \vee z \notin x)$. More precisely, the following theorem follows from Fact I.2.

Theorem I.3 There exists $\mathcal{V} \models \mathrm{Pt}+\operatorname{Refl}_{\Delta}+\operatorname{Subst}+\operatorname{Ext}_{\Delta}+\operatorname{Comp}\left[\mathscr{L}^{+}\right]$where $\operatorname{Refl}_{\Delta}: \equiv \forall x(x=x \leftrightarrow \Delta(x)), \operatorname{Ext}_{\Delta}: \equiv \forall x \forall y(\Delta(x) \wedge x=y \rightarrow x=y)$.

Proof Take $\mathcal{V}:=\widehat{U}$ with $U$ as in Fact I.2. By Lemma I.1 and what has been said above, we already know that $\mathcal{V} \models \mathrm{Pt}+\operatorname{Subst}+\operatorname{Comp}\left[\mathscr{L}^{+}\right]$. Now we observe that $\mathcal{U} \models \forall x \forall y(x \neq y \leftrightarrow((\nabla(x) \wedge \nabla(y)) \vee \neg(x=y)))$. Indeed, if $x \neq y$ and $x=y$, then $x \neq x$ and $y \neq y$, that is, $\nabla(x)$ and $\nabla(y)$; for the converse, if $\neg(x=y)$, then $x \neq y$ (because $U \models \mathrm{Pd}$ ), or if $x=y$ and $\nabla(x)$, then $x \neq y$ (because $U \models$ Subst), and so $x \neq y$. It follows that $\mathcal{V} \models \forall x \forall y(\neg(x=y) \leftrightarrow((\widehat{\nabla}(x) \wedge \widehat{\nabla}(y)) \vee x \neq y))$, or equivalently, that $\mathcal{V} \models \forall x \forall y(x=y \leftrightarrow((\Delta(x) \vee \Delta(y)) \wedge \neg(x \neq y))$, seeing that $\Delta(x) \equiv \neg \widehat{\nabla}(x)$. Therefrom, it is easy to see that, on the one hand, $\mathcal{V} \models \operatorname{Ext}_{\Delta}$ (considering that $\mathcal{V} \models \widehat{\mathrm{Ext}}$ ), and that, on the other hand, $\mathcal{V} \models \operatorname{Refl}_{\Delta}$ (using the fact that $\mathcal{U} \models$ Refl, and hence that $\mathcal{V} \models \forall x \neg(x \neq x))$.

So here is the first of the results announced in the Introduction proved. We now turn to the proof of the second one.

\section{Part II Topological Models}

Before proceeding to the description of the model we will be studying in the rest of this paper, we introduce a couple of notational conventions that will be useful.

Notation II.1 Given a function $f$, we use $f^{\prime} x$ for the pointwise image of $x$ if this is defined; otherwise, we write $\nexists f^{\prime} x$, and we use $f^{\prime \prime} X$ for the setwise image of $X$; that is, $f^{\prime \prime} X:=\left\{f^{\prime} x \mid x \in X\right\}$-which is always defined, thanks to the existence of $\varnothing$.

Notation II.2 Given an ordered pair $z=(x, y)$, we let $z^{+}$and $z^{-}$stand for $x$ and $y$, respectively.

Description The universe $|\mathcal{U}|$ of any $\mathscr{L}$-structure $\mathcal{U} \models$ Ext + Pt can be identified with a (proper!) subset of $F(|\mathcal{U}|)$, where $F(\cdot)$ is defined by

$$
F(X):=\{(A, B) \mid A, B \subseteq X \& A \cap B=\varnothing\} .
$$

The universe of our model will be obtained as inverse limit by iterating the operator $F(\cdot)$, as in [2], except that here, due to the nature itself of $F(\cdot)$, we will have to consider partial maps between consecutive levels; so we are led to define the notion of inverse limit for partial projective systems. The presentation we give is general enough to encompass the original construction given in [2] for the Pd case (which is sketched at the end of the paper).

The levels of the partial projective system $\left(U_{n+1} \stackrel{s}{\rightarrow} U_{n} \mid n \in \omega\right)$ we are interested in are defined by

$$
\left\{\begin{array}{l}
U_{0}:=\{\varnothing\} \\
U_{n+1}:=F\left(U_{n}\right), \text { for all } n \in \omega .
\end{array}\right.
$$


We then define the partial maps $s_{n+1}: U_{n+1} \rightarrow U_{n}$ inductively as follows:

$$
\begin{aligned}
& \text { Given }(A, B) \in U_{n+1} \text {, with } n \geqslant 1, \\
& s_{n+1}{ }^{`}(A, B):=\left(s_{n}{ }^{\prime} A, s_{n} \text { “' } B\right) \text { if this latter belongs to } U_{n} ; \\
& \quad \text { otherwise, } s_{n+1}{ }^{\circ}(A, B) \text { is not defined, that is, } \nexists s_{n+1}{ }^{\circ}(A, B) .
\end{aligned}
$$

And we take the following definition for $s_{1}$ (which is just a matter of choice):

$$
s_{1}{ }^{\prime}(\varnothing, \varnothing):=\varnothing \& \nexists s_{1}{ }^{\prime}(\{\varnothing\}, \varnothing), \nexists s_{1}{ }^{\prime}(\varnothing,\{\varnothing\}) .
$$

Note that, whatever the definition of $s_{1}$ is, $s_{n+1}: U_{n+1} \rightarrow U_{n}$ will always be partial for $n \geqslant 2 ;{ }^{5}$ this is due to the definition of $F(\cdot)$. Nevertheless, each $s_{n+1}: U_{n+1} \rightarrow U_{n}$ is surjective, and this is a key property for our construction to work. To see this, we inductively define a family of right inverses $i_{n}: U_{n} \rightarrow U_{n+1}$ by

$$
i_{0} ` \varnothing:=(\varnothing, \varnothing) \& i_{n+1}{ }^{\prime}(A, B):=\left(i_{n}{ }^{\prime} A, i_{n} “ B\right) \text {, for any }(A, B) \in U_{n} .
$$

It is easily seen by induction that each $i_{n}: U_{n} \rightarrow U_{n+1}$ so defined is indeed a total injective map such that $s_{n+1} \circ i_{n}=\delta_{U_{n}}$. From here on, we will let $s$ (respectively, $i$ ) stand for the union of the $s_{n} \mathrm{~s}$ (respectively, the $i_{n} \mathrm{~s}$ ). Then, by definition, we have $\left(s^{\natural} \xi\right)^{ \pm}=s^{\prime} \xi^{ \pm}$for all $\xi \in \bigcup_{n \geqslant 2} U_{n}$ such that $s^{\natural} \xi$ is defined, as well as $\left(i^{\prime} \xi\right)^{ \pm}=i^{\prime} \xi^{ \pm}$for all $\xi \in \bigcup_{n \geqslant 1} U_{n}$.

Sets in our model will be "s-sequences" (elements of $U_{\omega}$ defined below), and these are particular elements of the amalgamated Cartesian product:

$$
V:=\bigcup_{m \in \omega} V_{m}, \text { where } V_{m}:=\prod_{n \in \omega \backslash m} U_{n} .
$$

Thus, any element $u \in V$ is of the form $\left(u_{n}\right)_{n \geqslant m}$ with $u_{n} \in U_{n}$ for each $n \geqslant m$; we call $m$ the rank of $u$ and denote it by $\rho(u)$ (i.e., $\rho(u)$ is the unique $m$ for which $\left.u \in V_{m}\right)$. We will always be looking at $V$ as a topological space with the topology induced by the following notion of limit: $\lim _{k \rightarrow \infty} u^{k}=u$ if and only if for all $n \geqslant \rho(u)$, there exists $k_{n}$ such that for all $k \geqslant k_{n}, \rho\left(u^{k}\right) \leqslant n$ and $u_{n}^{k}=u_{n}$ (in other words, $\lim _{k \rightarrow \infty} u^{k}=u$ if and only if for each $n \geqslant \rho(u), \lim _{k \rightarrow \infty} u_{n}^{k}=u_{n}$ in $U_{n}$ equipped with the discrete topology).

Now we consider

$$
U_{\omega}=\lim _{\overleftarrow{n} \epsilon_{\omega}} U_{n}:=\left\{u \in V \mid \forall n \geqslant \rho(u), s^{`} u_{n+1}=u_{n} \& \nexists s^{`} u_{\rho(u)}\right\},
$$

which we thus define as the limit of the system $\left(U_{n+1} \stackrel{s}{\rightarrow} U_{n} \mid n \in \omega\right)$. We note that $U_{\omega}$ is a closed subset of $V$, but it is not a compact subspace. This will be clear under the next alternative presentation, which also shows that generalized projective limits as defined here are just particular subspaces of usual ones.

Take any object $\dagger \notin \bigcup_{n \geqslant 0} U_{n}$ and then, for each $n \in \omega$, let $\widetilde{U}_{n}:=U_{n} \cup\{\dagger\}$ and $\widetilde{s}_{n+1}$ be the extension of $s_{n+1}$ on $\widetilde{U}_{n+1}$ defined by $\widetilde{s}_{n+1}$ ' $\xi:=\dagger$ if $\nexists s_{n+1}$ ' $\xi$, and $\widetilde{s}_{n+1} \dagger:=\dagger$. We would define $\widetilde{i}_{n}$ accordingly. Again we let $\widetilde{s}$ (respectively, $\widetilde{i}$ ) stand for the union of the $\widetilde{s}_{n} \mathrm{~s}$ (respectively, the $\widetilde{i}_{n} \mathrm{~s}$ ).

Now we look at the limit of the projective system $\left(\widetilde{U}_{n+1} \stackrel{\widetilde{s}}{\rightarrow} \widetilde{U}_{n} \mid n \in \omega\right)$; that is,

$$
\widetilde{U}_{\omega}=\lim _{\bar{n} \widetilde{U}_{\omega}} \widetilde{U}_{n}:=\left\{a \in \widetilde{V}_{0} \mid \forall n \in \omega, \widetilde{s} a^{\prime} a_{n+1}=a_{n}\right\} \text { where } \widetilde{V}_{0}:=\prod_{n \in \omega} \widetilde{U}_{n},
$$

with the corresponding natural topology defined as above. 
The next fact, the proof of which is left to the reader, shows in particular that $\widetilde{U}_{\omega}$ is nothing but the Alexandroff compactification of $U_{\omega}$.

Fact II.3 $U_{\omega} \cong \widetilde{U}_{\omega} \backslash\left\{(\dagger)_{k} \geqslant 0\right\}$ and $X \subseteq U_{\omega}$ is compact if and only if $(\dagger)_{k} \geqslant 0 \notin \bar{X}$ in $\widetilde{U}_{\omega}$, if and only if $\{\rho(x) \mid x \in X\}$ is bounded in $\omega$.

In the sequel, we shall only look at $U_{\omega}$ as subspace of $V$. We will denote the projection of $U_{\omega}$ onto $U_{n}$ by $\pi_{n}$; that is, for all $u \in V, \pi_{n}{ }^{\prime} u:=u_{n}$ if $n \geqslant \rho(u)$, otherwise, $\nexists \pi_{n}{ }^{\prime} u$. By definition of $U_{\omega}, \pi_{n}$ ' $u=s \circ \pi_{n+1}$ ' $u$ for all $n \geqslant \rho(u)$.

As said, the universe of the $\mathscr{L}$-structure $U$ we are considering is $U_{\omega}$, in which we define the interpretations of the primitive symbols of $\mathscr{L}$ as follows:

$$
\left\{\begin{array}{l}
u \in_{\omega} v: \equiv \forall n \geqslant \max \{\rho(u), \rho(v)\}, u_{n} \in v_{n+1}^{+}, \\
u \notin_{\omega} v: \equiv \forall n \geqslant \max \{\rho(u), \rho(v)\}, u_{n} \in v_{n+1}^{-} ; \\
u=_{\omega} v: \equiv \rho(u)=\rho(v) \& \forall n \geqslant \rho(u), u_{n}=v_{n} ; \\
u \neq \neq_{\omega} v: \equiv \forall n \geqslant \max \{\rho(u), \rho(v)\}, \neg\left(u_{n}=v_{n}\right) .
\end{array}\right.
$$

It is easy to see that these binary relations thus defined are all closed subsets of $U_{\omega}^{2}$ and that they are such that $U \models \mathrm{Pt}$. Obviously, $={ }_{\omega}$ is nothing but the identity on $U_{\omega}$, so $U \models \mathrm{Eq}$ already. We are going to elaborate on $\neq_{\omega}$ subsequently. We start investigating the properties of $\mathcal{U}$ by showing it is extensional.

\section{Extensionality}

Lemma II.4 Given $u \in U_{\omega}, n+1 \geqslant \rho(u)$, and $\xi \in u_{n+1}^{+}$(respectively, $\xi \in u_{n+1}^{-}$), there exists $w \in U_{\omega}$ such that $w_{n}=\xi$ and $w \in_{\omega} u$ (respectively, $w \notin_{\omega} u$ ).

Proof By definition of $s$, given $j \geqslant 1$ and $\zeta \in u_{n+j}^{+}$, we may always find some $\zeta^{\prime} \in u_{n+j+1}^{+}$such that $s^{\prime} \zeta^{\prime}=\zeta$. Therefore, using the axiom of (dependent) choice, we can find a sequence $\xi^{k}, k \geqslant 0$, such that $\xi^{0}=\xi, \xi^{k} \in u_{n+k+1}^{+}$and $s^{\natural} \xi^{k+1}=\xi^{k}$ for all $k \geqslant 0$. We then take $w:=\left(\ldots, s^{`} s^{`} \xi^{0}, s^{`} \xi^{0}, \xi^{0}, \xi^{1}, \xi^{2}, \ldots\right)$. (The proof is similar with $\xi \in u_{n+1}^{-}$.)

\section{Proposition II.5 $\quad \mathcal{E} \models$ Ext.}

Proof Assume $u \models u=v$. Let $n+1 \geqslant \rho(u)$ and $\xi \in u_{n+1}^{+}$. By Lemma II.4, there exists $w \in U_{\omega}$ such that $w_{n}=\xi$ and $w \in_{\omega} u$. Then, by assumption, $w \in_{\omega} v$ too, so that $\xi=w_{n} \in v_{n+1}^{+}$. Hence, $u_{n+1}^{+} \subseteq v_{n+1}^{+}$for all $n+1 \geqslant \rho(u)$. Likewise, we show that $v_{n+1}^{+} \subseteq u_{n+1}^{+}$for all $n+1 \geqslant \rho(v)$, so that $u_{n+1}^{+}=v_{n+1}^{+}$for all $n+1 \geqslant \max \{\rho(u), \rho(v)\}$. In the same way, using Lemma II.4, we get $u_{n+1}^{-}=v_{n+1}^{-}$ for all $n+1 \geqslant \max \{\rho(u), \rho(v)\}$. It follows that $u_{n}=v_{n}$ for each $n \geqslant \rho(u)=\rho(v)$. Therefore, $u={ }_{\omega} v$; that is, $U \models u=v$.

We now turn to investigating the properties of $u$ with respect to comprehension. We will need several preliminary definitions and results. Let us first identify those elements of $F\left(U_{\omega}\right)$ that define sets in $u$.

Coded pairs We say that $\xi \in F\left(U_{\omega}\right)$ is coded in $\mathcal{U}$ if there exists $v \in U_{\omega}$ such that $\forall u \in U_{\omega}, u \in \xi^{+} \Leftrightarrow u \in_{\omega} v$ and $u \in \xi^{-} \Leftrightarrow u \notin_{\omega} v$. More generally, we will say that an ordered pair $\xi$ of subsets of $U_{\omega}$ is precoded in $U$ if there exists $v \in U_{\omega}$ such that $\forall u \in U_{\omega}, u \in \xi^{+} \Rightarrow u \in_{\omega} v$ and $u \in \xi^{-} \Rightarrow u \notin_{\omega} v$. Clearly, if $\xi$ is precoded, 
then $\xi \in F\left(U_{\omega}\right)$, but the converse is false. This should be clear in view of the next lemma.

We say that an ordered pair $\xi$ of subsets of $U_{\omega}$ is eventually partial if there exists $m \in \omega$ such that $\left(\pi_{n} \cdot \xi^{+}, \pi_{n}{ }^{*} \xi^{-}\right) \in U_{n+1}\left(=F\left(U_{n}\right)\right)$ for each $n \geqslant m .6$

Lemma II.6 $\xi$ is precoded in $\mathcal{U}$ if and only if $\xi$ is eventually partial.

Proof That $\xi$ is eventually partial if $\xi$ is precoded is obvious. Now, assuming $\xi$ is eventually partial, it is easily seen that $v \in U_{\omega}$ defined by $v_{n+1}:=\left(\pi_{n}{ }^{\prime} \xi^{+}, \pi_{n}{ }^{\prime} \xi^{-}\right)$ for each $n \geqslant n_{0}$, where $n_{0}$ is the least $n$ such that $\left(\pi_{n}\right.$ “ $\xi^{+}, \pi_{n}$ " $\left.\xi^{-}\right) \in U_{n+1}$ (so $\left.\rho(v)=n_{0}+1\right)$, is a precode of $\xi$ in $U$.

The following refinement is central to our investigations.

Proposition II.7 $\xi$ is coded in $\mathcal{U}$ if and only if $\xi$ is eventually partial and $\xi^{+}, \xi^{-}$ are closed subsets of $U_{\omega}$.

Proof It is easy to show that $\xi^{+}, \xi^{-}$are closed in $U_{\omega}$ provided $\xi$ is coded. Conversely, assume that $\xi$ is eventually partial and that $\xi^{+}, \xi^{-}$are closed in $U_{\omega}$, and then let $v \in U_{\omega}$ defined by $v_{n+1}:=\left(\pi_{n}\right.$ “' $\xi^{+}, \pi_{n}$ " $\left.\xi^{-}\right)$for each $n \geqslant n_{0}$, where $n_{0}$ is the least $n$ such that $\left(\pi_{n}{ }^{\prime} \xi^{+}, \pi_{n}{ }^{\prime} \xi^{-}\right) \in U_{n+1}$. We already know by the proof of the preceding lemma that $v$ is a precode of $\xi$. To see that $v$ is the code of $\xi$, let $u \in_{\omega} v$. So $u_{n} \in v_{n+1}^{+}=\pi_{n}{ }^{\prime} \xi^{+}$for all $n \geqslant \max \left\{\rho(u), n_{0}\right\}$. Then, for each $k \geqslant \max \left\{\rho(u), n_{0}\right\}$, choose $u^{k}$ in $\xi^{+}$such that $u_{k}^{k}=u_{k}$. Clearly, $\lim _{k \rightarrow \infty} u^{k}=u$, and as $\xi^{+}$is closed, we get $u \in \xi^{+}$. Likewise, one can show that $u \notin_{\omega} v$ implies $u \in \xi^{-}$.

So the main task is to look at those definable ordered pairs of subsets of $U_{\omega}$ that are both eventually partial and closed.

Partiality We say that a function $f: \omega^{N} \rightarrow \omega$ is progressive if $f$ is nondecreasing (i.e., $f^{\prime}\left(l_{0}, \ldots, l_{N-1}\right) \geqslant f^{\prime}\left(k_{0}, \ldots, k_{N-1}\right)$ whenever $l_{i} \geqslant k_{i}$ for each $i=0, \ldots, N-1)$ and $f^{\prime}\left(k_{0}, \ldots, k_{N-1}\right) \geqslant \max \left\{k_{0}, \ldots, k_{N-1}\right\}$.

Given two $(N+1)$-ary predicates $P(x, \bar{y})$ and $\bar{P}(x, \bar{y})$ on $U_{\omega}$, with $\bar{y}:=$ $y_{0}, \ldots, y_{N-1}$, we say that $(P, \bar{P})$ is uniformly eventually partial with respect to $x$ if there exists a progressive $f: \omega^{N} \rightarrow \omega$ such that for all $\bar{v}:=v_{0}, \ldots, v_{N-1}$ in $U_{\omega}$, for all $n \geqslant f^{\prime}\left(\rho\left(v_{0}\right), \ldots, \rho\left(v_{N-1}\right)\right)$, we have $\left(\left\{u_{n} \mid P(u, \bar{v})\right\},\left\{u_{n} \mid \bar{P}(u, \bar{v})\right\}\right) \in U_{n+1}$ (i.e., $\left.\left\{u_{n} \mid P(u, \bar{v})\right\} \cap\left\{u_{n} \mid \bar{P}(u, \bar{v})\right\}=\varnothing\right)$. Indication: the progressive function $f$ guarantees that the level $n$ at which $\left\{u_{n} \mid P(u, \bar{v})\right\}$ and $\left\{u_{n} \mid \bar{P}(u, \bar{v})\right\}$ become disjoint only depends on the rank of the parameters $\bar{v}$. For instance, it is easy to see that $\left(x \in_{\omega} y, x \notin_{\omega} y\right)$ and $\left(x==_{\omega} y, x \neq_{\omega} y\right)$ are both uniformly eventually partial with respect to $x$ and with respect to $y$.

Now, given an $(M+1)$-ary predicate $C(y, \bar{z})$ on $U_{\omega}$, with $\bar{z}:=z_{0}, \ldots, z_{M-1}$, we say that $C$ is uniformly compact with respect to $y$ if there exists a progressive function $g: \omega^{M} \rightarrow \omega$ such that for all $\bar{w}:=w_{0}, \ldots, w_{M-1}$ in $U_{\omega}$, we have $\rho(v) \leqslant g^{\prime}\left(\rho\left(w_{0}\right), \ldots, \rho\left(w_{M-1}\right)\right)$ for all $v$ in $U_{\omega}$ such that $C(v, \bar{w})$. Indication: the progressive function $g$ guarantees here that $\{\rho(v) \mid C(v, \bar{w})\}$ is bounded in $\omega$, so that $\{v \mid C(v, \bar{w})\}$ is compact in $U_{\omega}$ by Fact II.3 and that the corresponding bound only depends on the rank of the parameters $\bar{w}$. 
This last definition is motivated by the third point of the following result.

\section{Lemma II.8}

(i) If $(P, \bar{P})$ is uniformly eventually partial with respect to $x$, so is $(\bar{P}, P)$.

(ii) If $(P, \bar{P}) \&(Q, \bar{Q})$ are uniformly eventually partial with respect to $x$, so are $(P \wedge Q, \bar{P} \vee \bar{Q}) \&(P \vee Q, \bar{P} \wedge \bar{Q})$.

(iii) Suppose that $C$ is uniformly compact with respect to $y$ and that $x$ (distinct from $y)$ is not free in $C$. Then, if $(P, \bar{P})$ is uniformly eventually partial with respect to $x$, so are $\left(\forall_{C} y P, \exists_{C} y \bar{P}\right) \&\left(\exists_{C} y P, \forall_{C} y \bar{P}\right)$, where $\forall_{C} y X$ and $\exists_{C} y X$ are the abbreviations for $\forall y(C \rightarrow X)$ and $\exists y(C \wedge X)$, respectively.

Proof (i) is obvious, and (ii), (iii) are easily proved, so we may concentrate on establishing (iii). Let us write $C$ as $C(y, \bar{z})$ and $P$ as $P(x, \bar{y})$, where $\bar{y}:=y, \bar{z}$ and $\bar{z}:=z_{0}, \ldots, z_{M-1}$, and then, under the hypotheses, let $g: \omega^{M} \rightarrow \omega$ and $f: \omega^{M+1} \rightarrow \omega$ be progressive functions associated with $C$ and $P$, respectively, as in the definitions. We are going to show that $h: \omega^{M} \rightarrow \omega$ defined by $h^{\prime}\left(k_{0}, \ldots, k_{M-1}\right):=f^{\prime}\left(g^{\prime}\left(k_{0}, \ldots, k_{M-1}\right), k_{0}, \ldots, k_{M-1}\right)$ is a progressive function ensuring that $\left(\forall_{C} y P, \exists_{C} y \bar{P}\right)$ is uniformly eventually partial with respect to $x$. Being given $\bar{w}:=w_{0}, \ldots, w_{M-1}$ in $U_{\omega}$, consider $u, u^{\prime} \in U_{\omega}$ such that $\forall y(C(y, \bar{w}) \rightarrow P(u, y, \bar{w}))$ and $\exists y\left(C(y, \bar{w}) \wedge \bar{P}\left(u^{\prime}, y, \bar{w}\right)\right)$. Then choose $v \in U_{\omega}$ such that $\left.C(v, \bar{w}) \wedge \bar{P}\left(u^{\prime}, v, \bar{w}\right)\right)$, so that $\left.P(u, v, \bar{w})\right)$ as well. As $\rho(v) \leqslant g^{\prime}\left(\rho\left(w_{0}\right), \ldots, \rho\left(w_{M-1}\right)\right)$, we have $n \geqslant f^{\prime}\left(\rho(v), \rho\left(w_{0}\right), \ldots, \rho\left(w_{M-1}\right)\right)$ as soon as $n \geqslant h^{\prime}\left(\rho\left(w_{0}\right), \ldots, \rho\left(w_{M-1}\right)\right)$, so that $u_{n}$ and $u_{n}^{\prime}$ must be different for each $n \geqslant h^{\prime}\left(\rho\left(w_{0}\right), \ldots, \rho\left(w_{M-1}\right)\right)$. It follows that $\left\{u_{n} \mid \forall_{C} y P(u, y, \bar{w})\right\}$ and $\left\{u_{n} \mid \exists_{C} y \bar{P}(u, y, \bar{w})\right\}$ are disjoint for all $n \geqslant h^{`}\left(\rho\left(w_{0}\right), \ldots, \rho\left(w_{M-1}\right)\right)$. Now, that $\left(\exists_{C} y P, \forall_{C} y \bar{P}\right)$ is uniformly eventually partial with respect to $x$ too can be seen as a consequence of (i).

Continuity An $N$-ary predicate $P(\bar{x})$ on $U_{\omega}$ will be said to be continuous if and only if $\{(\bar{u}) \mid P(\bar{u})\}$ is closed in $U_{\omega}^{N}$. Examples of continuous (binary) predicates are given by $x \in_{\omega} y, x \notin_{\omega} y, x=\omega y, x \neq \omega y$.

Given an $(M+1)$-ary predicate $C(y, \bar{z})$ on $U_{\omega}$, with $\bar{z}:=z_{0}, \ldots, z_{M-1}$, we say that $C$ is approximable with respect to $y$ if, whenever $w_{i}=\lim _{k \rightarrow \infty} w_{i}^{k}$ in $U_{\omega}$, for each $i=0, \ldots, M-1$, and $C\left(v, w_{0}, \ldots, w_{M-1}\right)$ holds for some $v \in U_{\omega}$, one can find a sequence $v^{k}, k \in \omega$, in $U_{\omega}$ such that $\lim _{k \rightarrow \infty} v^{k}=v$ and $C\left(v^{k}, w_{0}^{k}, \ldots, w_{M-1}^{k}\right)$ holds for sufficiently large $k$. This technical definition will be seen at work in the proof of (ii) below.

\section{Lemma II.9}

(i) If $P \& Q$ are continuous, so are $P \wedge Q \& P \vee Q$.

(ii) Suppose that $C$ is approximable with respect to $y$. Then, if $P$ is continuous, so is $\forall_{C} y P$.

(iii) Suppose that $C$ is continuous and that $C$ is uniformly compact with respect to $y$. Then, if $P$ is continuous, so is $\exists_{C} y P$.

Proof (i) is obvious. For the proof of (ii), assume $P(x, y, \bar{z})$ is continuous and $C(x, y, \bar{z})$ is approximable with respect to $y$, with $\bar{z}:=z_{0}, \ldots, z_{M-1}$, and then consider converging sequences $u^{k}, w_{0}^{k}, \ldots, w_{M-1}^{k}, k \in \omega$, with respective limits $u, w_{0}, \ldots, w_{M-1}$ in $U_{\omega}$, such that $\forall y\left(C\left(u^{k}, y, w_{0}^{k}, \ldots, w_{M-1}^{k}\right) \rightarrow P\left(u^{k}, y, w_{0}^{k}, \ldots\right.\right.$, $\left.\left.w_{M-1}^{k}\right)\right)$ holds for all $k$. We have to show that $\forall y\left(C\left(u, y, w_{0}, \ldots, w_{M-1}\right) \rightarrow\right.$ 
$\left.P\left(u, y, w_{0}, \ldots, w_{M-1}\right)\right)$. So let $v$ in $U_{\omega}$ such that $C\left(u, v, w_{0}, \ldots, w_{M-1}\right)$. As $C$ is approximable with respect to $y$, there exists some sequence $v^{k}, k \in \omega$, in $U_{\omega}$ such that $\lim _{k \rightarrow \infty} v^{k}=v$ and $C\left(u^{k}, v^{k}, w_{0}^{k}, \ldots, w_{M-1}^{k}\right)$ holds for sufficiently large $k$, and so does $P\left(u^{k}, v^{k}, w_{0}^{k}, \ldots, w_{M-1}^{k}\right)$ by assumption. As $P$ is continuous, we thus get $\left.P\left(u, v, w_{0}, \ldots, w_{M-1}\right)\right)$, as expected.

For (iii), suppose that $P(x, y, \bar{z}) \& C(x, y, \bar{z})$ are continuous, with $\bar{z}:=z_{0}, \ldots$, $z_{M-1}$, and $C(x, y, \bar{z})$ is uniformly compact with respect to $y$; then consider converging sequences $u^{k}, w_{0}^{k}, \ldots, w_{M-1}^{k}, k \in \omega$, with respective limits $u, w_{0}, \ldots, w_{M-1}$ in $U_{\omega}$ such that $\exists y\left(C\left(u^{k}, y, w_{0}^{k}, \ldots, w_{M-1}^{k}\right) \wedge P\left(u^{k}, y, w_{0}^{k}, \ldots, w_{M-1}^{k}\right)\right)$ holds for all $k$. As $C$ is uniformly compact with respect to $y$, there exists a progressive function $g: \omega^{M+1} \rightarrow \omega$ such $\rho(v) \leqslant g^{\prime}\left(\rho\left(u^{k}\right), \rho\left(w_{0}^{k}\right), \ldots, \rho\left(w_{M-1}^{k}\right)\right)$ for each $v$ such that $C\left(u^{k}, v, w_{0}^{k}, \ldots, w_{M-1}^{k}\right)$. Choose $K \in \omega$ such that for all $k \geqslant K$, $\rho\left(u^{k}\right) \leqslant \rho(u) \& \rho\left(w_{0}^{k}\right) \leqslant \rho\left(w_{0}\right) \& \cdots \& \rho\left(w_{M-1}^{k}\right) \leqslant \rho\left(w_{M-1}\right)$. Thus, for each $k \geqslant K$, any $v$ in $U_{\omega}$ such that $C\left(u^{k}, v, w_{0}^{k}, \ldots, w_{M-1}^{k}\right) \wedge P\left(u^{k}, v, w_{0}^{k}, \ldots, w_{M-1}^{k}\right)$ will also be such that $\rho(v) \leqslant g^{\prime}\left(\rho(u), \rho\left(w_{0}\right), \ldots, \rho\left(w_{M-1}\right)\right)$. Let $v^{k}$ be one such $v$ for each $k \geqslant K$. So the sequence $v^{k}, k \geqslant K$, is inside $\left\{v \mid \rho(v) \leqslant g^{\natural}\left(\rho(u), \rho\left(w_{0}\right)\right.\right.$, $\left.\ldots, \rho\left(w_{M-1}\right)\right\}$, which is compact in $U_{\omega}$ by Fact II.3 (for any fixed $u, w_{0}, \ldots$, $w_{M-1}$ ); therefore, there exists a subsequence $v^{k_{j}}, j \in \omega$, that has a limit $v$ in $U_{\omega}$. As we have $C\left(u^{k_{j}}, v^{k_{j}}, w_{0}^{k_{j}}, \ldots, w_{M-1}^{k_{j}}\right) \wedge P\left(u^{k_{j}}, v^{k_{j}}, w_{0}^{k_{j}}, \ldots, w_{M-1}^{k_{j}}\right)$ for all $k_{j} \geqslant K$, we also get $C\left(u, v, w_{0}, \ldots, w_{M-1}\right) \wedge P\left(u, v, w_{0}, \ldots, w_{M-1}\right)$, because $C$ and $P$ are continuous. Thus, $\exists y\left(C\left(u, y, w_{0}, \ldots, w_{M-1}\right) \wedge P\left(u, y, w_{0}, \ldots, w_{M-1}\right)\right)$.

The result Let $\Phi(\bar{r}) \& \bar{\Phi}(\bar{r})$ be two abstract $N$-ary predicates, with $\bar{r}:=r_{0}, \ldots$, $r_{N-1}$, and let $\Theta_{s}(\bar{t})$ be an abstract $(M+1)$-ary predicate, in which we distinguish a free variable $s$ distinct from a given list $\bar{t}:=t_{0}, \ldots, t_{M-1}$ of other free variables. ${ }^{8}$ We define the language $\mathscr{L}_{\Phi, \bar{\Phi}, \Theta}^{+}$by means of the following rules:

(1) $\top$ and $\perp$ are formulas;

(2) If $x, y$ are variables, $x \in y$ and $x \notin y$ are formulas;

(3) If $x, y$ are variables, $x=y$ and $x \neq y$ are formulas;

(3) $)^{\prime}$ For any list $\bar{x}:=x_{0}, \ldots, x_{N-1}$ of variables, $\Phi(\bar{x})$ and $\bar{\Phi}(\bar{x})$ are formulas;

(4) If $\varphi, \psi$ are formulas, so are $\varphi \wedge \psi$ and $\varphi \vee \psi$;

$(5)^{\prime}$ If $\varphi$ is a formula and $x$ is a variable distinct from the free variables $\bar{t}$ of $\Theta_{S}(\bar{t})$, then $\forall_{\Theta} x \varphi$ and $\exists_{\Theta} x \varphi$ are formulas, to be understood as abbreviations for $\forall x\left(\Theta_{x}(\bar{t}) \rightarrow \varphi\right)$ and $\exists x\left(\Theta_{x}(\bar{t}) \wedge \varphi\right)$, respectively. ${ }^{9}$

Thereupon, we should add here the following rule, which is usually tacit or derivable in normal contexts:

(0) If $x, y$ are variables and $\varphi$ is a formula, so is $\varphi_{(y \mid x)}$, the result of substituting $y$ for some (not necessarily all) free occurrences of $x$ in $\varphi$.

Rule (0) allows us to change the names of the free variables of a formula $\varphi$ obtained from rule (5)' in order to apply again rule (5)' to $\varphi$ without unnecessary restrictionsas stated above, rule (5)' does not permit quantifications over one of the given free variables $\bar{t}$ of $\Theta_{s}(\bar{t})$ ! 
We then define the weak negation $\bar{\varphi}$ of an $\mathscr{L}_{\Phi, \bar{\Phi}, \Theta}^{+}$-formula $\varphi$ as in the Introduction, with here

and

$$
\overline{\Phi(\bar{x})} \text { is } \bar{\Phi}(\bar{x}), \quad \overline{\bar{\Phi}(\bar{x})} \text { is } \Phi(\bar{x}),
$$

$$
\overline{\forall_{\Theta} x \varphi} \text { is } \exists_{\Theta} x \bar{\varphi}, \quad \overline{\exists_{\Theta} x \varphi} \text { is } \forall_{\Theta} x \bar{\varphi} .
$$

We are now ready to state the second main result of this paper.

Theorem II.10 Let $\Phi_{\omega}(\bar{r}), \bar{\Phi}_{\omega}(\bar{r}), \Theta_{\omega}(s, \bar{t})$ be respective interpretations of $\Phi(\bar{r})$, $\bar{\Phi}(\bar{r}), \Theta_{s}(\bar{t})$ on $U_{\omega}$ such that $\Phi_{\omega}, \bar{\Phi}_{\omega}, \Theta_{\omega}$ are continuous, $\left(\Phi_{\omega}, \Phi_{\omega}\right)$ is uniformly eventually partial with respect to $r_{j}$ for all $j=0, \ldots, N-1$, and $\Theta_{\omega}$ is both uniformly compact with respect to $s$ and approximable with respect to $s$. Then $u \models \operatorname{Comp}\left[\mathscr{L}_{\Phi, \bar{\Phi}, \Theta}^{+}\right]$; that is,

$$
\mathcal{U} \models \forall \bar{z} \exists y \forall x((x \in y \leftrightarrow \varphi(x, \bar{z})) \wedge(x \notin y \leftrightarrow \bar{\varphi}(x, \bar{z}))),
$$

for any $\mathscr{L}_{\Phi, \bar{\Phi}, \Theta}^{+}$-formula $\varphi(x, \bar{z})$, with the proviso that the variable $x$ does not occur free within some occurrence of the predicate $\Theta .(\cdots)$ in $\varphi,{ }^{10}$ the variable $y$ is also supposed not to be free in $\varphi$, as usual.

Proof With any formula $\varphi(x, \bar{z})$ of $\mathscr{L}_{\Phi, \bar{\Phi}, \Theta}^{+}$we associate the predicate $P_{\varphi}(x, \bar{z})$ on $U_{\omega}$ defined by

$$
\text { for all } u, \bar{w} \text { in } U_{\omega}, P_{\varphi}(u, \bar{w}) \Leftrightarrow \mathcal{U}=\varphi(u, \bar{w}) .
$$

On the one hand, using the assumptions and Lemma II.8, one can show by induction on the complexity of $\varphi$ that $\left(P_{\varphi}, P_{\bar{\varphi}}\right)$ is uniformly eventually partial with respect to $x$ (the requirement on the variable $x$ is here justified for applying (iii) of Lemma II.8). On the other hand, using the assumptions and Lemma II.9, one can show by induction on the complexity of $\varphi$ that $P_{\varphi}$ (and so $P_{\bar{\varphi}}$ ) is continuous. It follows that, for any $\mathscr{L}_{\Phi, \bar{\Phi}, \Theta}^{+}$-formula $\varphi(x, \bar{z})$ (with the condition on $x$ ) and all $\bar{w}$ in $U_{\omega}$, the pair $\xi:=(\{u \mid \mathcal{U} \models \varphi(u, \bar{w})\},\{u \mid \mathcal{U} \models \bar{\varphi}(u, \bar{w})\})$ is eventually partial and $\xi^{+}, \xi^{-}$are closed subsets of $U_{\omega}$; so $\xi$ is coded in $U_{\omega}$ by Proposition II.7.

Theorem II.10 can naturally be extended to the case where are given several primitive predicates $\Phi, \bar{\Phi}, \Theta, \Phi^{\prime}, \bar{\Phi}^{\prime}, \Theta^{\prime}, \Phi^{\prime \prime}, \bar{\Phi}^{\prime \prime}, \Theta^{\prime \prime}$, and so on. We will now give three examples of natural and suitable $\Theta$ definable on $U_{\omega}$.

Example 1 Consider the preorder $\preccurlyeq \omega$ on $U_{\omega}$ defined by $u \preccurlyeq \omega v \Leftrightarrow \rho(u) \leqslant \rho(v)$ and the corresponding strict version $\prec_{\omega}$ defined by $u \prec_{\omega} v \Leftrightarrow \rho(u)<\rho(v)$. We denote the equivalence associated with $\preccurlyeq_{\omega}$ by $\sim_{\omega}$; that is, $u \sim_{\omega} v \Leftrightarrow \rho(u)=\rho(v)$. It is easy to see that all these binary relations define continuous predicates on $U_{\omega}$. It is not difficult either to show that $s \preccurlyeq \omega t, s \prec_{\omega} t$, and $s \sim_{\omega} t$ are all both uniformly compact with respect to $s$ and approximable with respect to $s$. By Theorem II.10, $u \models \operatorname{Comp}\left[\mathscr{L}_{\Theta}^{+}\right]$, where $\Theta_{\omega}(s, t): \equiv s R t$, with $R$ being any of $\preccurlyeq_{\omega}, \prec_{\omega}, \sim_{\omega}$.

Example 2 We here define a linear order $\leqslant_{\omega}$ on $U_{\omega}$ as follows. Take $\leqslant_{0}:=\{(\varnothing, \varnothing)\}$ on $U_{0}=\{\varnothing\}$ and then choose inductively any linear order $\leqslant_{n+1}$ on $U_{n+1}$, for each $n \in \omega$ so that the partial map $s_{n+1}: U_{n+1} \rightarrow U_{n}$ be order preserving, with $\xi<_{n+1} \zeta$ whenever $s_{n+1}$ ' $\xi$ is defined but $\nexists s_{n+1}$ ' $\zeta$. Now define $\leqslant \omega$ on $U_{\omega}$ by $u \leqslant \omega \quad v \Leftrightarrow \forall n \geqslant \max \{\rho(u), \rho(v)\}, u_{n} \leqslant n \quad v_{n}$. Note incidentally that $u \leqslant_{\omega} v \Rightarrow u \preccurlyeq_{\omega} v$ and $u \prec_{\omega} v \Rightarrow u<_{\omega} v$. Caveat: $\leqslant_{\omega}$ thus defined may not 
be a well-ordering on $U_{\omega}$, although each $\leqslant_{n}$ is one on $U_{n}$ (this latter being finite!). One can, however, show that $\leqslant_{\omega}$ is indeed a well-ordering when considered in $\mathcal{U}$ in the following sense: if $\varnothing \neq A \subseteq U_{\omega}$ and $A=\left\{u \mid u \in_{\omega} v\right\}$ for some $v \in U_{\omega}$, then $A$ has a least element for $\leqslant \omega \cdot{ }^{11}$ For our purposes, we notice that $\leqslant_{\omega}$ defines a continuous binary predicate on $U_{\omega}$, but not $<_{\omega}$. Nevertheless, if we define $<_{\omega}^{*}$ on $U_{\omega}$ by $u<_{\omega}^{*} v \Leftrightarrow u \leqslant \omega v \wedge u \neq_{\omega} v$, then $<_{\omega}^{*}$ is continuous. Then it is not hard to show that $s \leqslant \omega t$ and $s<_{\omega}^{*} t$ are also both uniformly compact with respect to $s$ and approximable with respect to $s$. By Theorem II.10, $U \models \operatorname{Comp}\left[\mathscr{L}_{\Theta}^{+}\right]$, where $\Theta_{\omega}(s, t): \equiv s R t$, with $R$ being $\leqslant_{\omega}$ or $<_{\omega}^{*}$.

Example 3 As refinement of $\sim_{\omega}$ above, the equivalence relation $\approx_{\omega}$ on $U_{\omega}$ defined by $u \approx_{\omega} v \Leftrightarrow\left(u \sim_{\omega} v \& u_{\rho(u)}=v_{\rho(v)}\right)$ is also easily seen to be suitable, so $u \models \operatorname{Comp}\left[\mathscr{L}_{\Theta}^{+}\right]$with $\Theta_{\omega}(s, t): \equiv s \approx_{\omega} t$. Note that $u \approx_{\omega} v \Leftrightarrow \neg\left(u \neq_{\omega} v\right)$, and we are going to have a closer look at $\neq_{\omega}$ precisely, as promised.

The inequality relation in $\mathcal{U}$ We point out some interesting properties of $\neq_{\omega}$ on $U_{\omega}$. The first one at least ensures that $\neq_{\omega}$ is not trivial - that is, $\neq_{\omega}$ is nonemptywhich should not be taken for granted in the Pt case!

Fact II.11 (nontriviality) $\quad \neq_{\omega}$ distinguishes infinitely many sets in $\mathcal{U}$.

Proof As noticed, $u \neq \neq_{\omega} v \Leftrightarrow \neg\left(u \approx_{\omega} v\right)$, and $\approx_{\omega}$ has infinitely many classes.

Now let $x \neq y: \equiv \exists z((x \in z \wedge y \notin z) \vee(x \notin z \wedge y \in z)){ }^{12}$ We have the following fact.

\section{Fact II.12 (upper separability) $\quad U \models \forall x \forall y(x \neq y \rightarrow x \neq y)$.}

Proof Suppose $u \neq \omega v$, and consider $w \in U_{\omega}$ defined by $w_{n+1}:=\left(\left\{u_{n}\right\},\left\{v_{n}\right\}\right)$ for sufficiently large $n$. Clearly, $u \in_{\omega} w$ and $v \notin_{\omega} w$.

Thereupon, we would just mention that $u \not \forall \forall x \forall y(x \neq y \rightarrow x \neq y)$ and that $\neq$ is not linked at all to $\neq$ in $\mathcal{U}$.

For the next property, let $\varnothing_{\omega}$ denote the code of $(\varnothing, \varnothing)$ in $U, \Lambda_{\omega}$ the one of $\left(\varnothing, U_{\omega}\right)$, and $\mathrm{V}_{\omega}$ the one of $\left(U_{\omega}, \varnothing\right)$. Note that $\rho\left(\varnothing_{\omega}\right)=0$, whereas $\rho\left(\Lambda_{\omega}\right)=$ $\rho\left(\mathrm{V}_{\omega}\right)=1 ; \Lambda_{\omega}$ and $\mathrm{V}_{\omega}$ are, respectively, the empty set and the universal set in the sense of $\mathcal{U}$.

Fact II.13 (hereditary separation) Suppose that $u \neq \neq_{\omega} \varnothing_{\omega} \& u \neq \neq_{\omega} \Lambda_{\omega}$ (respectively, $u \neq \omega \varnothing_{\omega} \& u \neq \neq_{\omega} \mathrm{V}_{\omega}$ ), and let $v \in U_{\omega}$ be such that $\forall w \in_{\omega} u, \forall w^{\prime} \in_{\omega} v$, $w \neq_{\omega} w^{\prime}$ (respectively, $\forall w \notin_{\omega} u, \forall w^{\prime} \notin_{\omega} v, w \neq_{\omega} w^{\prime}$ ). Then $u \neq_{\omega} v$.

Proof First, if $\rho(u)=0$, then obviously $u \approx_{\omega} \varnothing_{\omega}$. Hence $\rho(u) \geqslant 1$. Now it is easy to see that if $u_{\rho(u)}^{+}=\varnothing$, then $u \approx_{\omega} \varnothing_{\omega}$ or $u \approx_{\omega} \Lambda_{\omega}$ (respectively, if $u_{\rho(u)}^{-}=\varnothing$, then $u \approx_{\omega} \varnothing_{\omega}$ or $u \approx_{\omega} \mathrm{V}_{\omega}$ ). Therefore, if $u \neq \neq_{\omega} \varnothing_{\omega} \& u \neq \neq_{\omega} \Lambda_{\omega}$ (respectively, $u \neq \neq_{\omega} \varnothing_{\omega} \& u \neq \neq_{\omega} \mathrm{V}_{\omega}$ ), and we assume $u \approx_{\omega} v$, one can show by using Lemma II.4 that $\exists w \in_{\omega} u, \exists w^{\prime} \in_{\omega} v$ with $w \approx_{\omega} w^{\prime}$ (respectively, $\exists w \notin_{\omega} u, \exists w^{\prime} \notin_{\omega} v$ with $\left.w \approx_{\omega} w^{\prime}\right)$.

Fact II.14 (separation of complements) If $u \neq \neq_{\omega} \varnothing_{\omega}$, then $u \neq_{\omega} u^{c}$, where $u^{c}$ is the complement of $u$ in the sense of $U$; that is, $u^{c}$ is the code of $(A, B)$ in $U$ if and only if $u$ is the code of $(B, A)$ in $U$. 
Proof Assume $u \neq \neq_{\omega} \varnothing_{\omega}$, but $u \approx_{\omega} u^{c}$. Then, for $k:=\rho(u)=\rho\left(u^{c}\right)$, we have $u_{k}{ }^{+}=u_{k}^{c+}$ and $u_{k}{ }^{-}=u_{k}^{c-}$. But also, by definition of $u^{c}, u_{k}^{+}=u_{k}^{c-}$ and $u_{k}{ }^{-}=u_{k}^{c+}$. On the other hand, as $u \neq \neq_{\omega} \varnothing_{\omega}, u_{k}^{+} \cup u_{k}^{-}$should be nonempty, but $u_{k}^{+} \cup u_{k}^{-}=\left(u_{k}^{+} \cap u_{k}^{+}\right) \cup\left(u_{k}^{-} \cap u_{k}^{-}\right)=\left(u_{k}^{+} \cap u_{k}^{c+}\right) \cup\left(u_{k}^{-} \cap u_{k}^{c-}\right)=\left(u_{k}^{+} \cap u_{k}^{-}\right) \cup\left(u_{k}^{-} \cap u_{k}^{+}\right)=$ $\varnothing \cup \varnothing=\varnothing:$ a contradiction.

We shall end this paper with a remark clarifying the link between the construction given here and the one in [2].

The Pd case revisited Let us see what we get if we reiterate the construction described in Part II in the Pd case, that is, with $F(\cdot)$ defined by

$$
F(X):=\{(A, B) \mid A, B \subseteq X \& A \cup B=X\} .
$$

First of all, the maps $s_{n+1}$ of the projective system $\left(U_{n+1} \stackrel{s}{\rightarrow} U_{n} \mid n \in \omega\right)$ under consideration are now total maps, so that $U_{\omega}$ is simply a closed subset of $V_{0}=\prod_{n \in \omega} U_{n}$; that is, $\rho(u)=0$ for all $u \in U_{\omega}$. As $V_{0}$ is compact, so is $U_{\omega}$ now.

The definitions of $\epsilon_{\omega}, \notin_{\omega},=_{\omega}$ remain unchanged (with $\rho(u)=\rho(v)=0$ ), but we have to modify $\neq_{\omega}$ so that $U \models \mathrm{Pd}$. The definition of $\neq_{\omega}$ taken in [2] is $u \neq \neq_{\omega} v$ if and only if $u \models u \neq v$, which too gives a closed subset of $U_{\omega}^{2}$.

A corresponding notion of "eventually paradoxical" pairs is useless here as now it is easily seen that $\xi \in F\left(U_{\omega}\right)$ implies $\left(\pi_{n}{ }^{\prime} \xi^{+}, \pi_{n} " \xi^{-}\right) \in U_{n+1}$ for all $n$, so Proposition II.7 becomes " $\xi$ is coded in $\mathcal{U}$ if and only if $\xi^{+} \& \xi^{-}$are closed in $U_{\omega}$ only", and there is no analogue of Lemma II.8 here. On the other hand, Lemma II.9 is still of use, but we can dispense with requiring $C$ to be uniformly compact with respect to $y$ in clause (iii), for any closed subset of $U_{\omega}$ is compact here.

Examples of continuous predicates $\Theta_{\omega}(s, t)$ on $U_{\omega}$ that are approximable with respect to $s$ are now given by $s \in_{\omega} t \& s \notin_{\omega} t$. So Theorem II.10 yields $u \models \operatorname{Comp}\left[\mathscr{L}_{\Theta, \Theta^{\prime}}^{+}\right]$, where $\Theta_{\omega}(s, t): \equiv s \in_{\omega} t$ and $\Theta_{\omega}^{\prime}(s, t): \equiv s \notin_{\omega} t$, and that is the main result of [2], which we stated as Fact I.2 in this paper and used to prove Theorem I.3. We have thus come full circle.

\section{Conclusion}

What makes the usual technique of construction of models by inverse limits effective is the good behavior of positive (or more generally bounded positive) formulas with respect to the operations used in such constructions. In that regard, the paradoxical case is sensibly different and more appropriate than the partial one, in that the defining formula Pd is positive, whereas Pt is not. Indeed, as noticed above, Pd is preserved under projections, whereas Pt is not, which led us to consider generalized projective systems in this paper.

More generally, paradoxical pairs, that is, members of $\{(A, B) \mid A, B \subseteq U \&$ $A \cup B=U\}$, are preserved under topological closure on $U$, whereas partial ones, that is, members of $\{(A, B) \mid A, B \subseteq U \& A \cap B \neq \varnothing\}$, are not, which should introduce limitations for the Pt case, as in Theorem II.10. On the other hand, the topological interior operator does preserve partial pairs, by symmetry, so it would be more natural to think of partial sets in a topological model as partial open pairs. However, no such model could be normal ${ }^{13}$ (otherwise, each singleton would be open and the topology would be discrete), which also introduces limitations, as in 
Theorem I.3. So the present paper has somehow shown the limits of topological normal models in the Pt case.

\section{Notes}

1. Where $\varphi_{(y \mid x)}$ denotes any formula obtained from $\varphi$ by substituting $y$ for some - not necessarily all-free occurrences of $x$.

2. With that definition of $=$, the formulation of Ext here above may be referred to as the semantic version of extensionality.

3. We should remark that this is also related to the presence of an abstractor ' $\{\cdot \mid-\}$ ' in the language he considered, as explained in [3].

4. Given a set $X$, we let $\delta_{X}$ stand for $\{(x, x) \mid x \in X\}$.

5. With our definition $s_{2}$ is actually total!

6. It is easy to see that this amounts to only demanding that there exists some $n \in \omega$ such that $\left(\pi_{n} " \xi^{+}, \pi_{n}\right.$ “ $\left.\xi^{-}\right) \in U_{n+1}$.

7. Such a code is necessarily unique by Proposition II.5.

8. We would stress here that all those specified variables are actually definite concrete variables of the object language, not metavariables standing for variables, as the $x, y, \ldots$ in the rules below.

9. $\Theta_{x}(\bar{t})$ naturally stands for the result of substituting $x$ for $s$ in $\Theta_{s}(\bar{t})$.

10. That requirement will be justified in the proof.

11. A proof of this can be found in [4], where a similar construction is considered for the $\mathrm{Pd}$ case.

12. Compare with the definition of $x \neq y$ in Fact I.2.

13. By a normal model $U$, we mean one in which $=u$ is interpreted by the identity $\delta_{\mid} u \mid$.

\section{References}

[1] Gilmore, P. C., "The consistency of partial set theory without extensionality," pp. 147-53 in Axiomatic Set Theory, vol. 13, Part II of Proceedings of Symposia in Pure Mathematics, Los Angeles (1967), American Mathematical Society, Providence, 1974. Zbl 0309.02065. MR 0360271. 39, 40, 41

[2] Hinnion, R., "Naive set theory with extensionality in partial logic and in paradoxical logic,” Notre Dame Journal of Formal Logic, vol. 35 (1994), pp. 15-40. Zbl 0801.03019. MR 1271696. 41, 42, 43, 51 
[3] Libert, T., "Semantics for naive set theory in many-valued logics: Technique and historical account," pp. 121-36 in The Age of Alternative Logics. Assessing Philosophy of Logic and Mathematics Today, edited by J. van Benthem, G. Heinzmann, M. Rebuschi, and H. Visser, vol. 3 of Logic, Epistemology, and the Unity of Science, Springer, Dordrecht, 2006. Zbl 05180959. 39, 41, 52

[4] Libert, T., "ZF and the axiom of choice in some paraconsistent set theories," Logic and Logical Philosophy, (2003), pp. 91-114. Flemish-Polish Workshops II-IV and varia. Zbl 1117.03346. MR 2049085. 52

[5] Libert, T., "Models for a paraconsistent set theory," Journal of Applied Logic, vol. 3 (2005), pp. 15-41. Zbl 1063.03040. MR 2126452. 41, 42

Département de Mathématique Université Libre de Bruxelles CP211, Boulevard du Triomphe 1050 Brussels

BELGIUM

rhinnion@ulb.ac.be

tlibert@ulb.ac.be 